\section{Solution contact charging with respect to earthquake lights}

LOCKNER et al. ${ }^{1}$ have proposed a mechanism to explain the production of earthquake lights (EQLs) which strongly relies on the generation of electricity by the boiling of water in heated rocks in the shear zone. Using data from the original paper on saline charging ${ }^{2}$ and spray electrification they explain the coronal origin of EQLs for magnitudes 6-7 but were unable to account for those of the Matsushiro swarm. More recent investigations of solution contact charging ${ }^{3,4}$ have demonstrated that the charging mechanism is not as Blanchard originally speculated, that is, "mechanical pulverization of water" with charge values similar to those of spray electrification, but is a solidsolid contact mechanism. Whereas sprayed aqueous solution droplets carry charges of about $4 \times 10^{-19} \mathrm{C}$, those from solution contact boiling are in the range $3 \times 10^{-17}$ to $3 \times 10^{-14} \mathrm{C}$ (refs 4,5 ). The charges of $10^{-7} \mathrm{C} \mathrm{g}^{-1}$ quoted from Blanchard related to saline solution undergoing transition boiling on a surface at approximately $350^{\circ} \mathrm{C}$. However, at temperatures cited by Lockner et al., in excess of this, Leidenfrost boiling occurs to produce charges of $10^{-5} \mathrm{Cg}^{-1}$. Although Leidenfrost boiling produces fewer droplets, each carries higher charge by factors of 3-5 above those on sprayed droplets.

I suggest that the higher values are more likely and may also account for the EQLs of the Matsushiro swarm, thereby bringing the latter within compass of the proposed mechanism. One further observation is that previous studies of electrification from boiling have been based on $\mathrm{NaCl}_{\mathrm{aq}}$. An extension of these investigations ${ }^{6}$ has revealed that many aqueous solutions including samples of groundwaters will generate electricity by flash, transition and Leidenfrost boiling.

The more recent information would seem to strengthen the mechanism proposed for the production of EQLs though some modification is required in which spray electrification should be deleted where boiling is involved and the more suitable solution contact charging process substituted.

161 Cotmanhay Road,

\section{POUNDER}

Ilkeston,

Derbyshire, DE7 8QT, UK

\footnotetext{
1. Lockner, D. A., Johnston, M. J. S. \& Byerlee, J. D. Nature 302, 28-37 (1983)

2. Bianchard, D. C. Nature 201, 1164-1166 (1964).

3. Pounder, C. J. Electrostat. 1, 395-397 (1975)

4. Pounder, C. J. Electrostat. 9, 159-175; 177-182 (1980)

5. Bright, A. W. Proc. R. Instn Gt Br. 53, 267-269 (1981).

6. Pounder, C. Proc. Electrostat. '83, Inst. P. Ser. 66, 221-224 (1983).
}

\section{Carnivore social behaviour- does it need patches?}

RECENTLY, Macdonald ${ }^{1,2}$ presented an interesting model explaining the evolution of social behaviour in carnivores. Briefly, the model, called the resource dispersion hypothesis (RDH), is as follows. Important food for carnivores occurs in patches and food availability in these patches varies with, for example, weather and season. Frequently situations occur when only one of these patches yields the resources needed for the animals' maintenance. Such situations can occur during single nights or seasons. Hence, the carnivore has to include enough patches within its territory (see, for example, Fig. 1 of ref. 1) to reliably support its minimum needs in all such situations. Territory size and configuration is determined by "dispersion of transient patches of available prey (ephemeral from night to night, or shifting from one season to the next)" size, on the other hand, is positively correlated with the average patch quality (or quality of the least valuable patch, it is unclear which) (Fig. 1 of ref. 1).

Macdonald discusses predictions that are not necessarily borne out by the model, for example: "From the resource dispersion hypothesis one would not necessarily expect .... any relationship between group size and territory area, as the two are argued to be affected, largely independently, by the abundance and dispersion of available food respectively". The lack of such a correlation is then treated as a corroboration of the model ${ }^{1}$. However, as RDH cannot be falsified regardless of whether there is a correlation or not, this prediction is useless for testing the hypothesis. Here I will identify and evaluate one prediction that is an invariable consequence of RDH: when the temporal variation is such that access to a given patch is vital to the territory owners, then all group members are expected to exploit this patch simultaneously. If the group members regularly forage in different patches simultaneously then the basic assumption of ephemerally unique food patches falls and so does RDH. This point was stressed by Bradbury and Vehrencamp $^{3}$ when developing a model (very similar to RDH) to explain territory and group size in emballonurid bats. These authors ${ }^{3}$ found that all members of a given group of bats foraged in a common patch at any time.

Does it happen regularly that group members of, for example, badgers or red foxes forage simultaneously in a common patch? Macdonald's suggestion ${ }^{1}$ that it may be "on a bad night or a bad year" makes it difficult to evaluate this prediction. Individual badgers move and hunt solitarily inside a clan range $^{4}$ and individual foxes are in physical contact with one another for as little as $4 \mathrm{~min}$ each night $^{5}$. Also, Macdonald ${ }^{6}$ reported that dominant and subordinate red foxes in his study area outside Oxford generally foraged in different parts (and patches; Table 6 of ref. 6) of the group territory. Moreover, in the Revinge area in southern Sweden, breeding red foxes differed, in all seasons of the year, from their nonbreeding group members in their use of hunting habitat ${ }^{7}$. Thus, on neither a nightly nor a seasonal basis do members of a given group seem to be restricted in their hunting to a given patch. It is not likely that a whole group should unite at a single food patch during a 'bad' year, unless one considers the entire territory as one patch, but then RDH is no longer valid. Macdonald ${ }^{1}$ concludes that once group living in carnivores has evolved as an adaptation to resource dispersion then other benefits accruing from sociality per se (for example, cooperative hunting and defence of kills) further moulds the social organization in each population or species and that "the balance of these contemporary benefits is not necessarily the same as that which originally selected for group living". Unfortunately, this ad hoc argument makes every serious attempt to test RDH virtually impossible as each time data that would falsify the hypothesis are obtained, it could always be claimed that a particular population is no longer under the original selection pressure for sociality. Even so, this would not help either for the Oxford or for the Revinge red foxes, as both populations are believed to be at an early stage in the evolution of sociality ${ }^{1,8}$.

Recently ${ }^{8.9}$ I argued that permanently territorial altricial vertebrates that feed on an interannually fluctuating food resource, keep a constant territory size if the animals' lifespan is longer than the average time period of the food fluctuations. The animals' territory sizes will be so adjusted that they contain enough food for reproduction, or at least survival ${ }^{10}$, during the bottle-neck years of food scarcity. The model predicts that as food abundance increases, the territory owners, for example the breeding pair, take advantage of this resource surplus by allowing their adult offspring to remain at home, thereby increasing the group size. When food abundance decreases the subordinate group members will be evicted from the territory by the breeding pair. Hence, in any given territory of, say, foxes or badgers, group size will be positively correlated with the annual food abundance whereas territory size will be constant from year to year. This model does not include any assumption about ephemeral prey patches. A simple field test to distinguish 